\title{
A Rapid ELISA Method to Improve the Automated Test Throughput
}

Daniele Casini ${ }^{*}$, Paola Fontani ${ }^{1}$, Paolo Ruggiero', Enrico Balducci ${ }^{2}$ and Duccio Berti ${ }^{1}$

${ }^{1}$ GSK Vaccines S.r.I., Research Center, Siena, Italy

${ }^{2} \mathrm{School}$ of Biosciences and Veterinary Medicine, University of Camerino, Camerino, Italy

\begin{abstract}
In ELISA, ligand is commonly adsorbed to the plastic surface through non-covalent bonds between the hydrophobic regions of the ligand and the plastic surface. Thus, all the reactions occur in a heterogeneous phase, with some reactants in solution, and some immobilized. As a result, the diffusion constant of immobilized reagents is zero and the overall reaction speed is low. With the proposed new approach the first step occurs in a homogeneous phase, aimed at speeding up ELISA procedure especially to easily adapt it to robotic systems. Conventional tests usually may take up to 5 hours. Our "rapid ELISA" approach considerably reduces this time to less than 30 minutes allowing the method to be more suitable for automation.

The rapid ELISA has been set up to analyze samples coming from animal studies for vaccine development purposes. In particular, it was applied to quantitate antibodies specific for the Outer Membrane Vesicle of Neisseria meningitidis group B and Influenza virus antigens (H1N1; H3N2; B). Due to its high flexibility, this rapid ELISA can be used to detect a wide range of antibodies raised against a wide range of antigens.
\end{abstract}

Keywords: ELISA; OMV; Homogeneous phase; Heterogeneous phase; Immune complex; ALP-conjugated antibody

\section{Introduction}

Enzyme-linked immunosorbent assay (ELISA) [1-5] is one of the most commonly used methods for the detection and quantitation of antibodies, antigens, hormones, and a large variety of other molecules, including synthetic peptides. Indeed, ELISA is extremely popular both in research and in clinical studies also because it is relatively inexpensive compared with other more sophisticated technologies. The most common procedure can be summarized as it follows: a ligand (very often an antigen, antibody or peptide) is coated onto the surface of each well in micro plates. The residual, non-coated area of the well is then saturated with an inert protein or polymer to prevent non-specific binding of the subsequent reagents. Subsequently, the molecule to be quantified is dispensed in the plate allowing the binding to the ligand. Finally, a specific enzyme-conjugated antibody, followed by a specific substrate, originates a colored reaction product that is proportional to the amount of the bound molecule under investigation and can be quantified by photometry.

In ELISA all the reactions occur in a heterogeneous phase, because some reagents are in solution, while other ones are adsorbed and immobilized. As a result the diffusion constant of the immobilized reagent is zero and the overall reaction speed is very low. For this reason the incubation times are normally very long, lasting usually hours.

Here, we describe a rapid and easy method to considerably speed up ELISA protocol, based on the transformation of the step in which serum antibodies and secondary conjugated antibodies react from heterogeneous to homogeneous phase reaction. All the data herein reported to optimize this approach refer to an ELISA aimed at detecting total immunoglobulin level in mouse sera against the Outer Membrane Vesicles (OMV) of Neisseria meningitidis group B [6-8] but it could be potentially applied to any other antigen. This assay was developed to analyze samples coming from animal studies for vaccine development purposes, thus it can be specifically recommended for vaccine immunogenicity studies.

\section{Materials and Methods}

\section{Plate preparation}

The antigen Outer Membrane Vescicles (OMV) was diluted in tris(hydroxymethyl)aminomethane (TRIS) buffer $(\mathrm{pH} 9.0)$ to a concentration of $5.0 \mu \mathrm{g} / \mathrm{ml}$ (expressed as protein concentration). 100 $\mu \mathrm{l}$ aliquots of this solution were dispensed into each well of 96-well microtiter polystyrene plates (Maxisorp Nunc/Thermo; Roskilde, Denmark). After an incubation of $2.5 \mathrm{~h}$ at $30^{\circ} \mathrm{C}$, plates were washed with $350 \mu \mathrm{l} /$ well of washing buffer, [10 mM phosphate buffer and $150 \mathrm{mM}$ $\mathrm{NaCl}$ (pH 7.4)] (PBS) containing $0.05 \%$ (vol/vol) Tween 20 . Wells were then saturated with $250 \mu \mathrm{l}$ of a $3 \%$ (wt/vol) Polyvinylpyrrolidone-15 (PVP) in distilled water and incubate for $1 \mathrm{~h}$ at room temperature (22$25^{\circ} \mathrm{C}$ ). Plates are then washed 3 times with washing buffer as described above.

\section{Conventional ELISA method}

Each plate contained a reference mouse serum, represented by a pool of sera from mice immunized with OMV vaccine and a quality control serum, represented by another pool of immunized mouse sera. To the reference serum was assigned a titer of $5000 \mathrm{U} / \mathrm{ml}(\mathrm{Units} / \mathrm{ml})$ (this titer represents the reciprocal of dilution giving A $405 \mathrm{~nm}=2.5 \mathrm{in}$ this assay). Each serum was diluted in dilution buffer [PBS containing $0.05 \%$ ( $\mathrm{vol} / \mathrm{vol}$ ) Tween 20 , and $2 \%(\mathrm{wt} / \mathrm{vol}$ ) of bovine serum albumin]. Reference serum was diluted in duplicate starting from a dilution 1:3,000 and then following a two-fold dilution along the strips. Quality

*Corresponding author: Daniele Casini, GSK Vaccines S.r.I., Research Center via Fiorentina 1, 53100 Siena, Italy, Tel: +39 057724 3227; Fax: +39 0577 278600; E-mail: daniele.x.casini@gsk.com

Received October 07, 2015; Accepted November 09, 2015; Published November 16, 2015

Citation: Casini D, Fontani P, Ruggiero P, Balducci E, Berti D (2015) A Rapid ELISA Method to Improve the Automated Test Throughput. J Anal Bioanal Tech S13: 005. doi:10.4172/2155-9872.S13-005

Copyright: @ 2015 Casini D, et al. This is an open-access article distributed under the terms of the Creative Commons Attribution License, which permits unrestricted use, distribution, and reproduction in any medium, provided the original author and source are credited. 
control serum and sera to be tested were diluted in the same way as reference serum. Before plate dispensing, all dilutions were prepared in test tubes or low binding plates (Masterblock; Greiner Bio-One, Germany) or tubes. After sample dispensing, plates were incubated for $2 \mathrm{~h}$ at $37^{\circ} \mathrm{C}$. Then they were washed with washing buffer. Afterwards $100 \mu$ of goat anti-mouse IgG ( $\gamma$-chain specific) conjugated to alkaline phosphatase (Sigma Chemical Co., St. Louis, MO, USA) diluted $1: 10,000$ in PBS-BSA buffer were added to each plate well. Plates were incubated for $2 \mathrm{~h}$ at $30^{\circ} \mathrm{C}$ and washed as already described. A chromogenic substrate solution was prepared at the concentration of $1 \mathrm{mg} / \mathrm{ml}$ dissolving $\mathrm{p}$-Nitrophenyl Phosphate (p-NPP) tablets (Sigma Chemical Co., St. Louis, MO, USA) in 1.0M Diethanolamine buffer pH 9.8 containing $0.5 \mathrm{mM} \mathrm{MgCl}_{2}$ (Sigma Chemical Co., St. Louis, $\mathrm{MO}$, USA). Finally $100 \mu$ of the chromogenic solution were dispensed into each well. After $30 \mathrm{~min}$ at room temperature the reaction was stopped with $100 \mu \mathrm{l}$ of $4 \mathrm{~N} \mathrm{NaOH}$, and plates were read at $405 \mathrm{~nm}$.

\section{Rapid ELISA procedure}

The same reagents and procedures used in conventional method were utilized for plate coating and plate saturation. The plate was prepared as for the conventional ELISA with the exception that samples, reference and quality control sera were diluted in dilution buffer containing 1:1,000 goat anti-mouse IgG ( $\gamma$-chain specific) conjugated to alkaline phosphatase (ALP), allowing homogeneous phase reaction. The time needed for this step ("pre-incubation") is 5 minutes; longer time of pre-incubation was assessed. $100 \mu \mathrm{l}$ of diluted sample were immediately dispensed into the plates (final dilution 1:6000). As for conventional method all sera were then diluted along the strips following a two-fold dilution step. Plates were incubated for 20 minutes at room temperature. This incubation time was chosen because in preliminary setting-up of the assay increasing time up to $30^{\prime}$ decreased linearity of the results, while shorter incubation time resulted in too low signal (not shown). After incubation, plates were washed as described for the conventional method. The chromogenic substrate p-NPP solution was prepared according to the procedure already described but at the concentration of $3.0 \mathrm{mg} / \mathrm{ml}$ and $100 \mu \mathrm{l}$ aliquots of this solution were dispensed into each well. After 10 minutes at room temperature the reaction was stopped with $100 \mu \mathrm{l}$ of $4 \mathrm{~N} \mathrm{NaOH}$ and plates read at $405 \mathrm{~nm}$.

\section{Data reduction and statistical analysis}

Non-parametric test were applied. To compare recoveries obtained with different serum dilutions Mann-Whitney test was used (Figure 1). To evaluate the correlation between rapid and conventional method Spearman test was used (Figure 2). All the sera were tested at different dilutions and the corresponding absorbance values at $405 \mathrm{~nm}$ were interpolated on the regression line obtained from absorbance values and titers at different dilutions of the reference serum [8].

\section{Results}

\section{Optimization of pre-incubation time and serum dilution}

As shown in Figure 1A increasing pre-incubation time did not influence the results, thus the assay was carried out with 5 minutes pre-incubation. In order to determine the performances of the assay several serum dilutions were investigated. As shown in Figure 1B and $1 \mathrm{D}$ the final dilution of sera which gave the best result, i.e., the one closest to $100 \%$ recovery, was 1:6,000. As defined in FDA Guidance for Industry, Bioanalytical Method Validation Guidelines [9], the recovery is the detector response obtained from an amount of the analyte (serum) added to and extracted from the biological matrix (negative serum), compared with the detector response obtained for the true concentration of the pure authentic standard. Recovery pertains to the extraction efficiency of an analytical method within the limits of variability. The non-extracted standards represent $100 \%$ recovery (with suggested acceptance range between 85 and 115\% [9]). The percentage of recovery of the values with known titer samples was utilized as an indicator to choose the best serum dilution.

\section{Optimization of the dilution of the Alkaline Phosphatase (ALP) conjugated antibody}

As already stated, the serum samples to be tested were diluted 1:3,000 in a buffer containing the Alkaline Phosphatase (ALP) conjugated antibody. This step was performed in a test tube, allowing the reaction between the ALP-conjugated antibody and the immunoglobulins present in the serum occurring very rapidly in a homogeneous phase. A series of different dilutions of the ALP-conjugated antibody were performed in order to identify the best performances in terms of accuracy of the test (recovery \%): the dilution 1:1,000 was found to be the closest to $100 \%$ recovery, and thus was used in the study (Figure $1 \mathrm{C)}$.

\section{Optimization of the dilution of the color developing solution}

The concentration of p-Nitrophenyl Phosphate in the substrate solution was tested starting from $1.0 \mathrm{mg} / \mathrm{ml}$ through $4.0 \mathrm{mg} / \mathrm{ml}$, at steps of $1.0 \mathrm{mg} / \mathrm{ml}$. The experimental conditions were: Serum dilution 1:6,000; anti-mouse antibody ALP conjugated 1:1,000; incubation time of the homogeneous phase 10 minutes; incubation time of the immune complex 30 minutes at room temperature (RT). We observed that the assay linearity clearly improved by increasing p-NPP concentration, with an optimum at $3.0 \mathrm{mg} / \mathrm{ml}$ (data not shown).

\section{Data from rapid and conventional methods highly correlate}

Figure 2A shows the high correlation observed between rapid and conventional method to detect antibody against OMV of Neisseria meningitidis group B in sera from 18 immunized mice. All titers are expressed as arbitrary ELISA units $/ \mathrm{ml}(\mathrm{EU} / \mathrm{ml})$. The titration has been performed against a reference serum to which an arbitrary value was assigned.

\section{Rapid ELISA outperforms conventional ELISA when short incubation times are applied}

The conditions used for rapid ELISA protocol (secondary $\mathrm{Ab}$ concentration, serum dilution, incubation times) were also applied in parallel to a conventional ELISA method using reference serum against FLU antigen (A/California/07/2009, 20,000 EU/ml) in order to compare the results of the two methods and to better define the relevance of the change of the first incubation step from heterogeneous (conventional ELISA) to homogeneous (rapid ELISA) phase, which in this case represented the only difference between the two methods. Results in Figure $2 \mathrm{C}$ show a very low signal obtained with the conventional method compared with the rapid one.

To compare the background signal obtained with the two methods, a positive control and a negative control (unrelated serum) were tested with rapid and conventional methods at the same dilution. By conventional ELISA, positive control gave $\mathrm{A}_{405}=1.721 \pm 0.087$ (SD) $(\mathrm{N}=8)$, negative control gave $\mathrm{A}_{405}=0.082 \pm 0.010(\mathrm{~N}=12)$, with an $\mathrm{S} / \mathrm{N}$ ratio $=21.0$; by rapid ELISA, the corresponding values were $2.512 \pm$ $0.170(\mathrm{~N}=8), 0.125 \pm 0.029(\mathrm{~N}=12)$, and 20.1 respectively, with very little difference, in particular for $\mathrm{S} / \mathrm{N}$ ratio. 
A

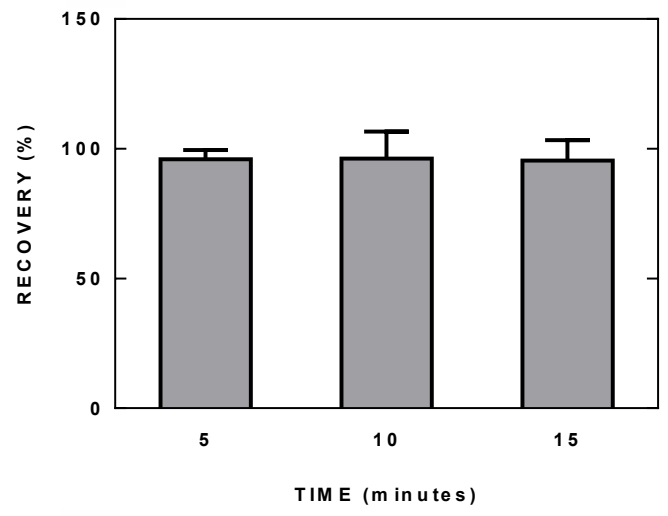

C



ALP CONJUGATED ANTIBODIES DILUTION
B



D

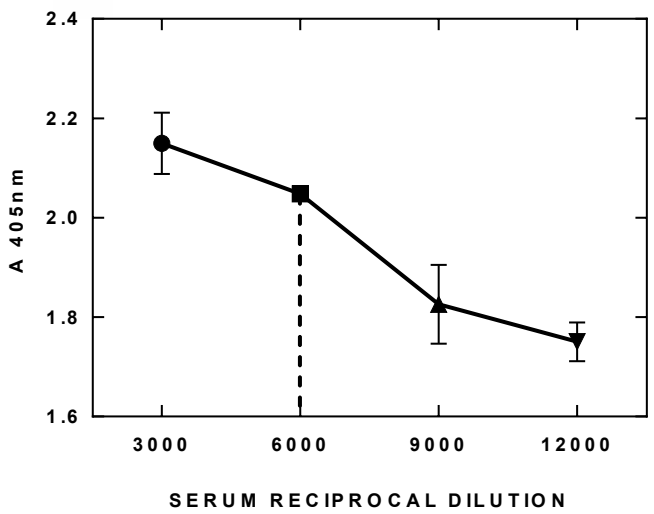

Figure 1: Summary of the optimization of the assay conditions. (A) No differences in $\%$ of recovery were obtained by increasing pre-incubation time of the immune-complex; $N=4$. (B) The percentage of recovery vs. serum reciprocal dilution indicates that the serum dilution $1: 6,000$ is the one closest to $100 \%$. $P$ values are reported on the top. (C) The result closest to $100 \%$ recovery is obtained using a dilution of 1:1,000 of the ALP-conjugated antibody. (D) The sensitivity of the assay vs. serum reciprocal dilutions; $\mathrm{N}=3$. The serum dilution of 1:6,000, selected because it resulted in the recovery closest to $100 \%$, gives a good sensitivity.

\section{Discussion}

The rapid ELISA procedure offers the possibility of speeding up ELISA in a perspective of applying this method to a robotic system. In particular, serum antibodies to be detected and labelled secondary antibodies are allowed to react in a test tube. As each reactant is in solution, the equilibrium of the reaction (antibody-antigen binding) is reached very quickly. Moreover, the use of pre-formed immunecomplex allows in turn shortening also the subsequent steps, and makes unnecessary the washings between the incubation with the primary and the secondary antibody, needed in the conventional ELISA.

Although the optimization study has been carried out only for OMV of Neisseria meningitidis group B, it is applicable to other antigens against which antibody titer has to be determined, as demonstrated by preliminary results obtained with FLU antigens (Figure $2 \mathrm{C}$ ), and in principle to any kind of ELISA, in particular for immunogenicity studies. However, it must be pointed out that, changing the antigen/ antibody to be assessed, the sensitivity and specificity should be carefully evaluated and the method validated case by case.

\section{Conclusion}

In summary, as schematized in Figure 2D, the proposed method for rapid ELISA comprises three steps after plate preparation. First, the specific antibody reacts with secondary antibody in a homogeneous, liquid phase. Second, the pre-formed immune complex (specific antibodies and secondary antibodies) binds to the specific antigen coated to the plate. Third, the chromogenic substrate is added and the color-generating reaction occurs.

The considerable reduction of incubation time of the immune complexes (20 minutes) as compared with conventional procedure (approximately 2 hours plus 2 hours of secondary antibodies incubation) is due to the pre-formed immune complexes. In other words, in the conventional procedure, a large amount of serum antibodies must be attached to the coated plates to give reasonable chances to the secondary labeled antibody "to find" and bind them in a heterogeneous system during a reasonable time.

In the proposed rapid ELISA, the vast majority of specific serum antibodies were already bound by secondary antibodies in the homogenous phase and at a higher concentration of secondary conjugated antibody compared with conventional ELISA. Each immune complex then will bind to the plate producing an effective signal, because it already carries an ALP molecule which will hydrolyze the substrate producing a photometric signal. This method, requiring 
Citation: Casini D, Fontani P, Ruggiero P, Balducci E, Berti D (2015) A Rapid ELISA Method to Improve the Automated Test Throughput. J Anal Bioanal Tech S13: 005. doi:10.4172/2155-9872.S13-005

A

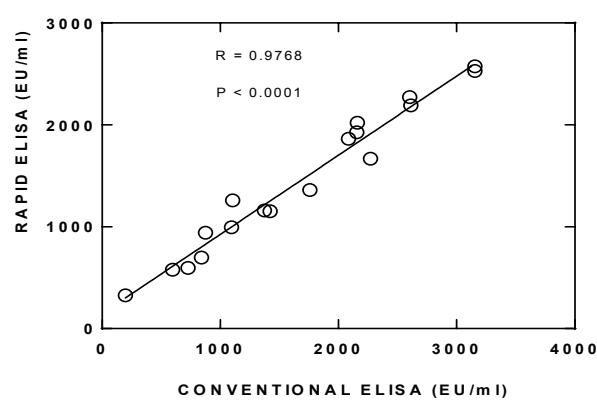

B

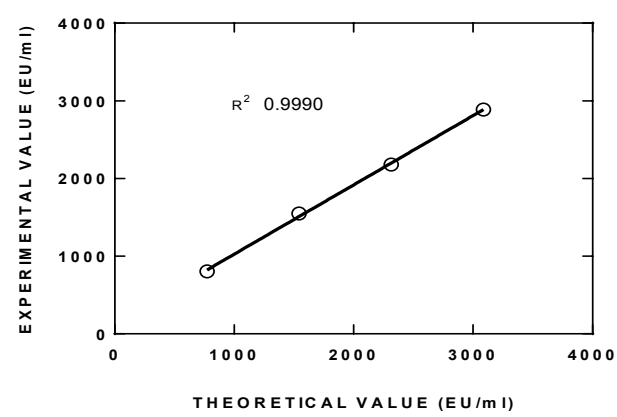

C

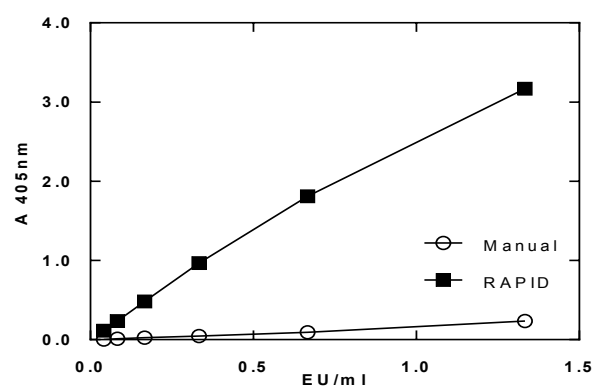

D

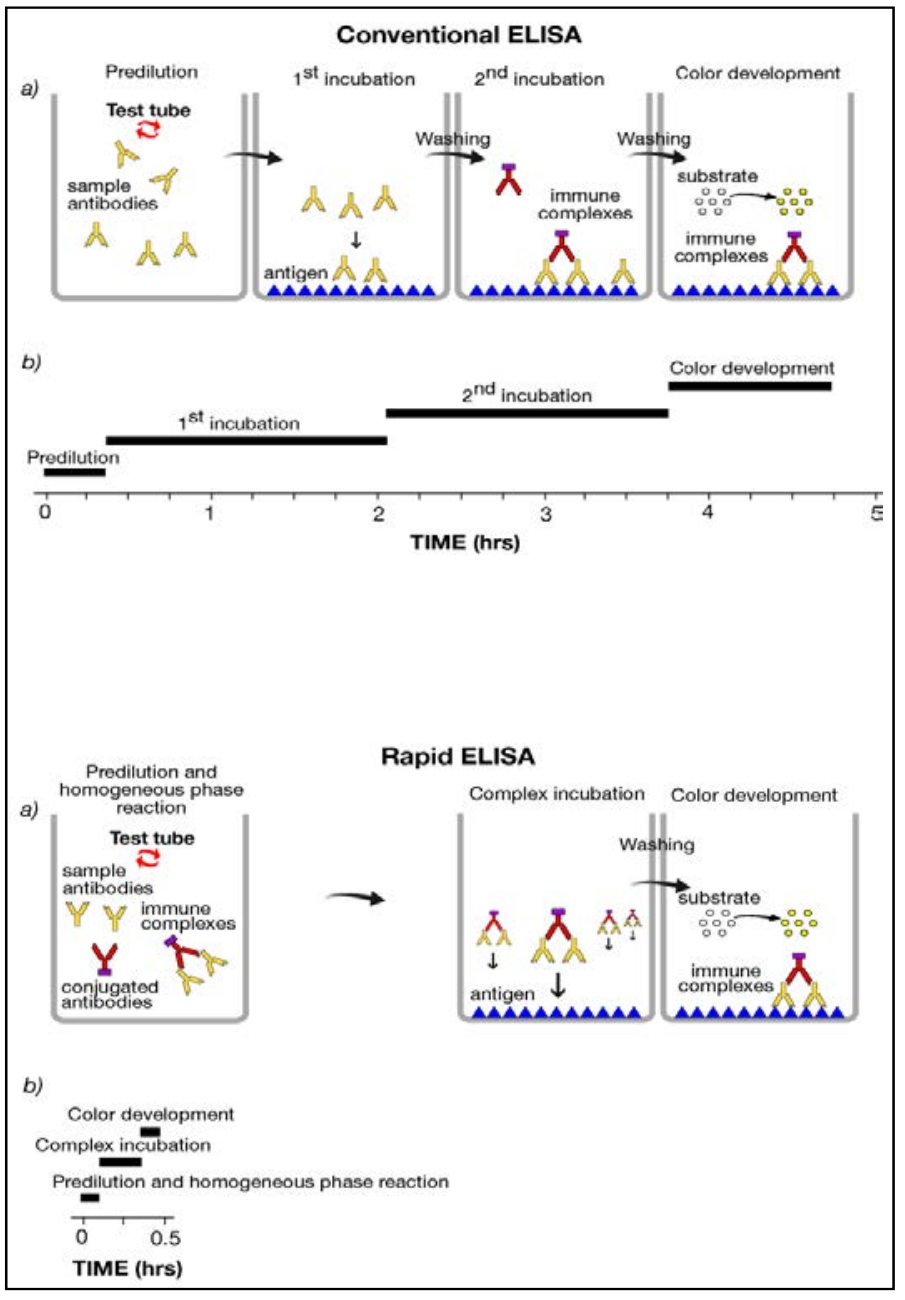

Figure 2: Comparison between conventional and rapid ELISA. (A) In both axes are reported the ELISA units per $\mathrm{ml}$ (EU/ml) obtained for a given sample with each of the methods. The two methods displays high correlation $(R=0.9768) ; N=18$. (B) In the linear range of the assay, the values of $E U / m l$ measured by rapid ELISA ( $Y$ axis) show good correspondence with the known values ( $X$ axis). (C) Comparison of results obtained with 2-fold serial dilutions of an anti-FLU A/ California/07/2009 reference serum diluted starting from 1:15,000 by carrying out both rapid and conventional ELISA using the incubation times of the rapid. (D) Schematic representation of the rapid ELISA in comparison with the conventional one.

only brief contact between the immobilized antigens and the sample, eliminates long incubation times. This allows specific affinity capture at $37^{\circ} \mathrm{C}$ while minimizing non-specific binding and matrix effects, which can compromise the performance of traditional ELISAs. It is also important to emphasize that this method is very simple, inexpensive and straightforward since no sophisticated reagents are required. The considerable shortening of the entire procedure is extremely useful for high throughput applications and has been successfully implemented to high-throughput ELISA in our laboratories.

\section{Acknowledgements}

This method has been patented by the authors as reported below: US Patent Serial: 12/084,358, Application number: 20100035285 European Patent Office: Application no. 06847258.8-2404, Patent No. PCT/IB2006004004

\section{References}

1. Engvall E, Perlmann P (1971) Enzyme-linked immunosorbent assay (ELISA) Quantitative assay of immunoglobulin G. Immunochemistry 8: 871-874.
2. Magar E (1972) Data Analysis in Bioch and Bioph. Academy Press, New York.

3. Lequin RM (2005) Enzyme immunoassay (EIA)/enzyme-linked immunosorbent assay (ELISA). Clin Chem 51: 2415-2418.

4. Avrameas S, Guilbert B (1971) Enzymo-immunological determination of proteins with the aid of immunoadsorbants and enzyme-labelled antigens. CR Acad Sci Hebd Seances Acad Sci D 273: 2705-2707.

5. Schuurs AHWM, van Weemen BK (1982) Method for the determination of antigens and antibodies. US Patent 3654090.

6. Rappuoli R (2001) Conjugates and reverse vaccinology to eliminate bacterial meningitis. Vaccine 19: 2319-2322.

7. Schuchat A, Robinson K, Wenger JD, Harrison LH, Farley M, et al. (1997) Bacterial meningitis in the United States in 1995. Active Surveillance Team. N Engl J Med 337: 970-976.

8. Pizza M, Scarlato V, Masignani V, Giuliani MM, Aricò B, et al. (2000) Identification of vaccine candidates against serogroup $B$ meningococcus by whole-genome sequencing. Science 287: 1816-1820.

9. US Department of Health and Human Services, Food and Drug Administration (2001) Guidance for Industry, Bioanalytical Method Validation. 